\title{
The price of defence: toxins, visual signals and oxidative state in an aposematic butterfly
}

\author{
Jonathan D. Blount ${ }^{{ }^{* \dagger} \dagger}$, Hannah M. Rowland ${ }^{2,3 \dagger}$, Christopher Mitchell ${ }^{1}$, Michael \\ P. Speed ${ }^{4}$, Graeme D. Ruxton ${ }^{5}$, John A. Endler ${ }^{6}$, Lincoln P. Brower ${ }^{7}$ \\ ${ }^{1}$ Centre for Ecology \& Conservation, College of Life \& Environmental Sciences, University \\ of Exeter, Penryn Campus, Cornwall TR10 9FE, UK
}

${ }^{2}$ Max Planck Institute for Chemical Ecology, Hans-Knöll-Straße 8, Jena, 07745, Germany

${ }^{3}$ Department of Zoology, University of Cambridge, Cambridge CB2 3EJ, UK

${ }^{4}$ Institute of Integrative Biology, University of Liverpool, Liverpool L69 7ZB, UK

${ }^{5}$ School of Biology, Sir Harold Mitchell Building, Greenside Place, St Andrews, United Kingdom

${ }^{6}$ Centre for Integrative Ecology, School of Life \& Environmental Sciences, Deakin University, Waurn Ponds VIC 3216, Australia

${ }^{7}$ Department of Biology, Sweet Briar College, Sweet Briar VA 24595, USA

* Authors for correspondence: j.d.blount@exeter.ac.uk and hrowland@ice.mpg.de

$\dagger$ Joint first authors

Running headline: Oxidative stress and warning signal honesty 


\section{SUMMARY}

2 In a variety of aposematic species, the conspicuousness of an individual's warning signal and

3 the quantity of its chemical defence are positively correlated. This apparent honest signalling

4 in aposematism is predicted by resource competition models which assume that the

5 production and maintenance of aposematic defences compete for access to antioxidant

6 molecules that have dual functions as pigments directly responsible for colouration and in

7 protecting against oxidative lipid damage. Here we study a model aposematic system - the

8 monarch butterfly (Danaus plexippus) and make use of the variable phytochemistry of its

9 larval host-plant, milkweeds (Asclepiadaceae), to manipulate the concentration of

10 sequestered cardenolides. We test two fundamental assumptions of resource competition

11 models: that (1) the possession of secondary defences is associated with costs in the form of

12 oxidative lipid damage and reduced antioxidant defences; and (2) that oxidative damage or

13 decreases in antioxidant defences can reduce the capacity of individuals to produce

14 aposematic displays. Monarch caterpillars that sequestered the highest concentrations of

15 cardenolides exhibited higher levels of oxidative lipid damage as adults. The relationship

16 between warning signals, cardenolide concentrations and oxidative damage differed between

17 the sexes. In male monarchs conspicuousness was explained by an interaction between

18 oxidative damage and sequestration: as males sequester more cardenolides, those with high

19 levels of oxidative damage become less conspicuous, while those that sequester lower levels

20 of cardenolides equally invest in conspicuous with increasing oxidative damage. There was

21 no significant effect of oxidative damage or concentration of sequestered cardenolides on

22 female conspicuousness. Our results demonstrate physiological linkage between the

23 production of coloration and protection from autotoxicity, that warning signals can be honest

24 indicators of defensive capability, and that the relationships are different between the sexes. 
25 Key-words: aposematism, cardenolides, cardiac glycosides, resource competition, oxidative

26 lipid damage, monarch butterfly, honest signalling. 


\section{Introduction}

Many species of animals, plants and micro-organisms possess chemical defences that reduce the likelihood of their being eaten [1]. Aposematic species, such as the monarch butterfly (Danaus plexippus), signal their chemical defences to predators with conspicuous warning colours [2]. In some species, the conspicuousness of the warning signal and the quantity of chemical defence are positively correlated, for example in dendrobatid frogs [3-5], marine opisthobranchs [6], ladybird beetles [7, 8], and paper wasps Polistes dominula [9]. The identification of these relationships has led to renewed interest in the idea that warning signals may inform predators about levels of prey defence and that such signals are honest indicators of defensive capability [10]. Several theoretical studies have, however, predicted the opposite: that well defended prey should reduce investment in signals, because signalling carries an additional conspicuousness cost, and such prey stand a good chance of surviving any attack unharmed [11-13]. Negative signal-defence correlations have been reported across Dendrobatid species of poison dart frogs [14] including the granular poison dart frog Dendrobates granuliferus [15]. Using a novel theoretical model Blount et al [16] could explain both the positive and negative correlations between warning colours and chemical defences if these two traits are linked through the competitive use of a shared resource. One possible shared resource is energy, which can be limiting for the sequestration or biosynthesis of toxins [17]. Alternatively, sequestration of toxins could impose metabolic costs in terms of oxidative lipid damage and reduced antioxidant defences - an idea that has has received

47 limited empirical attention in regard to warning signals.

Many plant allelochemicals are powerful pro-oxidants, which, when ingested, can cause oxidative lipid damage [18]. In their resource competition framework, Blount et al [16] envisaged that pigments used in prey warning signals (e.g., carotenoids, flavonoids, melanins, and pteridines) might play a dual role imparting colour as well as acting as antioxidants that 
prevent self-damage when storing toxins. In their model, if antioxidants are required to enable high levels of toxicity, signal reliability can be explained if the brightest and most toxic species gain access to more of the limiting resource than those that are less bright and less toxic. The prediction of signal honesty breaks down in their model when prey have very abundant resources, in which case it pays to divert these resources increasingly into toxins and not into warning signals, because less conspicuous but highly toxic prey encounter predators less often and have high chances of surviving attacks [16]. Because directly controlling antioxidant availability is challenging experimentally, whether colour-toxin relationships are influenced by the resource state of prey is poorly understood $[14,15]$, and has not yet been examined experimentally $[11,12,16,17,19]$.

Here we manipulate diet-derived toxins available to an individual to test whether toxin sequestration and the production and maintenance of warning signals are linked via oxidative state. We achieve this by using a model aposematic system, the monarch butterfly, that selectively metabolises cardenolides present in its host-plants and stores them in its body [20, 21], and by using the known inter- and intraspecific variability in cardenolide concentration and cardenolide polarity of the monarch's larval milkweed host-plants (Asclepiadaceae; [21, 22]) to manipulate the toxins available to an individual to sequester. We test whether (1) the quantity of sequestered secondary defence by caterpillars is associated with oxidative lipid damage and reduced antioxidant defences in adults; and (2) whether oxidative lipid damage incurred during sequestration affects the capacity of adult butterflies to produce aposematic displays.

Because there is substantial genetic variation in sequestration ability, and because evolutionary history and contemporary species interactions may influence patterns of cardenolide sequestration [23] we used monarchs from the same population. We reared them 
on four milkweed species that show within- and between-species, and among-population variation in cardenolide concentration, diversity, and polarity [24-27]. The polarity of cardenolides can influence their toxicity [21] and transport [28], so our four plant species were used to represent a gradient of distinct cardenolide defence, with the aim to create different costs of sequestration.

We reared monarch caterpillars on either Ascelpias curassavica, which is a critical hostplant worldwide and contains a diversity of cardenolides, including apolar voruscharin which is detrimental to monarch performance [21, 29-32]; A. syriaca (common milkweed) which is the principal native food plant of the monarch east of the Rocky Mountains and is characterized by more polar cardenolides though also contains the apolar labriformin [33, 34]; A. incarnata (swamp milkweed) which is a preferred hostplant in the Midwest USA and has low concentrations of cardenolides [35]; or A. tuberosa (butterfly milkweed) which is an occasional native foodplant in the wild with low concentrations of cardenolides $[21,36]$.

Based on the published records of biological activity and chromatic quantification (e.g., HPLC and thin layer chromatography) of cardenolides of milkweeds in different populations/latitudes [37, 38], we predicted that concentrations of cardenolides in the body and wings of newly eclosed adults would differ according to the cardenolides of the hostplant on which they were reared (see methods): A. curassavica $(A c) \geq A$. syriaca $(A s)>A$. incarnata $(A i) \geq A$. tuberosa $(A t)$. Because sequestration, modification, and storage of allelochemicals are believed to be oxidatively stressful for many chemically defended organisms [18], we predicted that body levels of oxidative lipid peroxidation would differ amongst individuals according to host-plant, as follows: $A c \geq A s>A i \geq A t$. In turn, we predicted that survival to eclosion would differ according to host-plant as follows: $A t \geq A i>A s \geq A c$, and that the redness, luminance, and conspicuousness of the warning signals would differ: 
101 individual levels of cardiac glycosides (CGs), oxidative state, and warning signal redness,

102 luminance, and conspicuousness. We predicted a positive association between levels of CGs

103 and and lipid peroxidation, a negative association of CGs with antioxidant defences, and that

104 highly toxic prey will reduce investment in signals if they have high levels of oxidative

105 damage because they cannot bear the oxidative cost of investing in both traits [16].

106 Materials and methods

107 (a) Milkweed plants

108 Milkweeds (A. incarnata, A. tuberosa, A. curassavica, and A. syriaca) were locally sourced

109 in Amherst, Virginia, USA. We did not measure cardenolide content in the leaves of the

110 plants used in the experiment, but used published records about the spatial repeatability of

111 cardenolide defences that take into account latitudinal clines [37-39]. From these records we

112 predicted that for milkweeds grown in Mid-Atlantic and Southeastern regions of the United

113 States, total cardenolide content would vary in the order A. curassavica $(A c) \geq A$. syriaca $(A s)$

$114>A$. incarnata $(A i) \geq A$. tuberosa $(A t)$. The plants were grown without the use of pesticides as

115 described previously [40]. All plants were healthy with undamaged leaves at the start of the

116 experiment.

117 (b) Milkweed Feeding Assay and preparation of samples

118 Eggs were obtained from wild caught Monarch females in Virginia (USA) and larvae were

119 reared by LPB on single plants. All larvae had abundant food during the experiment. Larvae

120 were monitored daily for pupation starting at Day 12.

121 Upon eclosion, the date and the individual's sex were recorded. Adults were placed in

122 glassine envelopes and frozen on dry ice until dead, then stored in a freezer at $-80^{\circ} \mathrm{C}$ before

123 being shipped to the UK on dry ice. Adults were stored at $-80^{\circ} \mathrm{C}$ in JDB's laboratory until

124 they were analysed. 
125 For analysis, the gut was removed, and the head, thorax and abdomen (hereafter, 'body')

126 were bisected longitudinally. One half of the body was returned to storage at $-80^{\circ} \mathrm{C}$ for later

127 analysis of cardenolides. The other half of the body was immediately homogenised in ice-

128 cold phosphate-buffered saline (PBS; $5 \% \mathrm{w} / \mathrm{v})$, for analyses of oxidative stress. The wings

129 were removed using dissecting scissors and shipped on dry ice to HMR's laboratory for

130 analyses of colouration (see below). Wings were then returned (on dry ice) to JDB's

131 laboratory for cardenolide analysis.

\section{(c) Determination of oxidative stress and cardenolide concentration}

133 Determining oxidative state requires a range of assays including antioxidant defences and

134 oxidative damage. We assayed three such components: total superoxide dismutase (SOD),

135 which are metalloenzymes that catalyse the dismutation of superoxide into oxygen and

136 hydrogen peroxide and form a crucial part of intracellular antioxidant defences; total

137 antioxidant capacity (TAC), which measures the activity of low molecular weight chain-

138 breaking antioxidants including ascorbate, a-tocopherol, carotenoids and flavonoids, and

139 therefore gives an overview of the antioxidant status of individuals (including diet-derived

140 antioxidants); and malondialdehyde (MDA), which is formed by the $\beta$-scission of peroxidised

141 polyunsaturated fatty acids, and therefore is a definitive marker of oxidative damage.

(i) Total superoxide dismutase (SOD)

143 Total SOD was assayed by measuring the dismutation of superoxide radicals generated by

144 xanthine oxidase and hypoxanthine. We followed the instructions of the kit (\#706002;

145 Cayman Chemical, Michigan, USA), except that tissue homogenates were further diluted

146 with the supplied sample buffer $(1: 100 \mathrm{v} / \mathrm{v})$ to ensure that SOD activity fell within the range

147 of the standard curve. Samples were assayed in duplicate and are reported as units of SOD

148 activity (U) per mg tissue. 
150 TAC was assayed by measuring the capacity of tissue homogenate to inhibit the oxidation of 2,2'-azino-di-[3-ethylbenzthiazoline sulphonate] (\#709001; Cayman Chemical). The homogenate was further diluted with sample buffer $(1: 5 \mathrm{v} / \mathrm{v})$ to bring the absorbance values within the range of the standard curve. Samples were assayed in duplicate, as per the kit instructions. Data are reported as nmols of TAC activity (Trolox equivalents) per g tissue.

MDA was measured using HPLC with fluorescence detection as described previously for tissue homogenates (e.g. Blount \& Pike 2011). All chemicals were HPLC grade, and chemical solutions were prepared using ultra pure water (Milli-Q Synthesis; Millipore,

159 Watford, UK). Samples were derived in $2 \mathrm{~mL}$ capacity polypropylene screw-top

160 microcentrifuge tubes. For tissue homogenates and for the standard $(1,1,3,3-$ tetraethoxypropane, TEP; see below) a $20 \mu \mathrm{l}$ aliquot was added to $20 \mu 1$ butylated hydroxytoluene solution (BHT; 0.05\% w/v in $95 \%$ ethanol), $160 \mu 1$ phosphoric acid solution $\left(\mathrm{H}_{3} \mathrm{PO}_{4} ; 0.44 M\right)$, and $40 \mu$ thiobarbituric acid solution (TBA; $\left.42 \mathrm{mM}\right)$. Samples were capped, vortex mixed for $5 \mathrm{~s}$, then heated at $100^{\circ} \mathrm{C}$ for $1 \mathrm{~h}$ in a dry bath incubator to allow formation of MDA-TBA adducts. Samples were then cooled on ice for $5 \mathrm{~min}$, before $80 \mu 1 n$ butanol was added and tubes were vortex mixed for $20 \mathrm{~s}$. Tubes were centrifuged at $12 \mathrm{x} \mathrm{g}$ and $4{ }^{\circ} \mathrm{C}$ for $3 \mathrm{~min}$, before the upper ( $n$-butanol) phase was collected and transferred to an HPLC vial for analysis. Samples $(10 \mu 1)$ were injected into a Dionex HPLC system (Dionex

169 Corporation, California, USA) fitted with a $5 \mu \mathrm{m}$ ODS guard column and a Hewlett-Packard

170 Hypersil $5 \mu$ ODS $100 \times 4.6 \mathrm{~mm}$ column maintained at $37^{\circ} \mathrm{C}$. The mobile phase was methanol-buffer (40:60, v/v), the buffer being a $50 \mathrm{~m} M$ anhydrous solution of potassium monobasic phosphate at $\mathrm{pH} 6.8$ (adjusted using $5 \mathrm{M}$ potassium hydroxide solution), running isocratically over $3.5 \mathrm{~min}$ at a flow rate of $1 \mathrm{ml} \mathrm{min}^{-1}$. Data were collected using a 
174

175

176

177

178

179

180

181

182

183

184

185

186

187

188

189

190

191

192

193

194

195

196

197

198

fluorescence detector (RF2000; Dionex) set at $515 \mathrm{~nm}$ (excitation) and $553 \mathrm{~nm}$ (emission).

For calibration a standard curve was prepared using a TEP stock solution ( $5 \mathrm{~m} M$ in $40 \%$ ethanol) serially diluted using $40 \%$ ethanol. Data are presented as nmols MDA per g tissue.

\section{(iv) Cardenolide analysis}

For cardenolide analysis, samples (individual wings and the remaining half of the body) were dried in an oven at $60{ }^{\circ} \mathrm{C}$ for $16 \mathrm{~h}$. The dried material was ground to a fine powder using a pestle and mortar, and weighed to the nearest $0.0001 \mathrm{~g}$ using a GR-202 electronic balance (A\&D Instruments Ltd., Abington, UK). The samples were placed in screw-top polypropylene tubes for de-fatting and cardenolide extraction. To remove fats, $2 \mathrm{ml}$ hexane was added and samples were incubated at $35^{\circ} \mathrm{C}$ for $30 \mathrm{~min}$, before being centrifuged at $10 \mathrm{x} \mathrm{g}$ and $4^{\circ} \mathrm{C}$ for $5 \mathrm{~min}$; the supernatant was collected and discarded. Ethanol (1.9 ml) containing $20 \mu \mathrm{g}$ digitoxin (\#D5878; Sigma-Aldrich, Dorset, UK) as an internal standard were added to the pellet and it was briefly vortexed to extract cardenolides (Ritland 1994). Samples were placed in an XUB5 ultrasonic bath (Grant Instruments, Shepreth, UK) at $60^{\circ} \mathrm{C}$ for $60 \mathrm{~min}$, followed by further centrifugation $\left(9.6 \mathrm{xg}\right.$ and $4^{\circ} \mathrm{C}$ for $\left.5 \mathrm{~min}\right)$. The supernatant was removed and the remaining pellet dried using a Savant ISS 110 SpeedVac Concentrator at room temperature (Thermo Scientific, Altrincham, UK). Samples were then dissolved in $0.5 \mathrm{ml}$ methanol by vortexing, and $50 \mu \mathrm{l}$ of the solution was injected into a Dionex HPLC system fitted with a Waters Spherisorb $5 \mu \mathrm{m}$ ODS2 column $(150 \times 4.6 \mathrm{~mm})$ maintained at $20^{\circ} \mathrm{C}$. A multistep gradient of acetonitrile $(\mathrm{ACN})$ and ultra pure water was used as the mobile phase, as follows: initial concentration $20 \% \mathrm{ACN}$ held for 5 minutes, ramp to $70 \% \mathrm{ACN}$ at 20 mins and held until 25 minutes, ramp to $95 \% \mathrm{ACN}$ at 30 mins and held until 35 mins, returning to

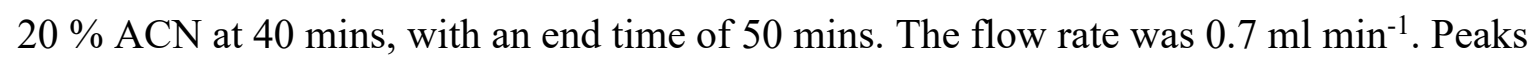
were collected using a PDA-100 photodiode array detector (Dionex) at an absorbance value of $218 \mathrm{~nm}$. Spectral data between $200-400 \mathrm{~nm}$ were collected, and cardenolides were 
199 identified as peaks with a symmetrical absorbance band with a maximum absorbance

200 between $217-222 \mathrm{~nm}[41,42]$. Data are presented as $\mu \mathrm{g}$ total cardenolides per $0.1 \mathrm{~g}$ tissue.

(d) Measurements and visual modelling of colouration

202 The reflectance of the fore- and hindwings of each butterfly, and of four leaves from one of

203 the representative backgrounds of the leaves of Asclepias syriaca (obtained from A.

204 Agrawal), were measured using an Ocean Optics USB2000 spectrophotometer, with

205 specimens illuminated at $45^{\circ}$ to normal by a DH1000 balanced halogen deuterium light

206 source. The measuring spot diameter was $3 \mathrm{~mm}$, with spectra recorded at $0.34 \mathrm{~nm}$ intervals

207 from 300 to $700 \mathrm{~nm}$ and measured relative to a WS-1 reflectance standard. For each wing, 3-4

208 non-overlapping measurements were taken per orange segment of the wing (figure s1; total

209 number of measurement spots per forewing $=13-14$; hind wing $=28-29$ ). For the leaves we

210 took ten non-overlapping measurements. Spectrophotometry data were recorded using Ocean

211 Optics OOBase32.

212 Reflectance spectra were reduced to $1 \mathrm{~nm}$ intervals ranging from wavelengths $300-700 \mathrm{~nm}$

213 using customised MATLAB code (written by I. C. Cuthill). To determine differences in

214 chroma between treatments, we modelled the predicted photon catches of a generalized

215 passerine bird (passerines being the main avian predators of monarchs [43]) for each

216 spectrum following the Vorobyev-Osorio model [44], using a customized MATLAB code

217 (provided by I. C. Cuthill, 2006). We used the cone types of a blue tit, Cyanistes caeruleus

218 [45, longwave, LWS, $\lambda \max 563 \mathrm{~nm}$; mediumwave, MWS, $\lambda \max 503 \mathrm{~nm}$; shortwave, SWS,

$219 \lambda \max 448 \mathrm{~nm}$; ultraviolet, UVS, $\lambda \max 371 \mathrm{~nm}$; and double dorsal, DD $\lambda \max 563 \mathrm{~nm}$;

$22046]$.We calculated redness as the ratio of long wave to medium wave cone responses. We also

221 calculated a measure of conspicuousness as $\Delta \mathrm{S}$ between the butterflies and $A$. syriaca leaves

222 using the Vorobyev-Osorio colour discrimination model, which is based on evidence that

223 colour discrimination is determined by noise arising in the photoreceptors and is independent 
of light intensity. We used a Weber fraction value of 0.05 for the most abundant cone type

[47]. We calculated luminance as the response of the dorsal double cone [48].

\section{(e) Data analyses}

227 Left- and right-wing cardenolide concentration (from here on CG) were significantly

228 positively correlated $(\mathrm{r}=0.96, \mathrm{t}=20.75, \mathrm{df}=38, \mathrm{p}=2.2 \mathrm{e}-16)$. There was no significant

229 correlation between asymmetry in wing CG concentrations and the mean CG of the wings (r

$230=0.10, \mathrm{t}=0.64, \mathrm{df}=38, \mathrm{P}=0.52)$, and so for each individual we calculated a mean wing $\mathrm{CG}$

231 concentration. Left- and right-wing redness were significantly positive correlated $(\mathrm{r}=0.65, \mathrm{t}$

$232=5.30, \mathrm{df}=38, \mathrm{P}=5.2 \mathrm{e}-06)$, as were left- and right-wing conspicuousness $(\mathrm{r}=0.59, \mathrm{t}=4.41$, $233 \mathrm{df}=37, \mathrm{P}=8.6 \mathrm{e}-05)$, and left- and right-wing luminance $(\mathrm{r}=0.396, \mathrm{t}=2.66, \mathrm{df}=38, \mathrm{P}=$

234 0.0114). For each individual we calculated a mean forewing redness, conspicuousness, and

235 luminance.

236 We log transformed malondialdehyde (MDA) and wing and body CG and to normalise the

237 distribution of the residuals. We analysed the effect of foodplant on total CG content,

238 bodyCG and wingCG content using a GLM with a Gaussian distribution and identity link

239 function. We set $A$. tuberosa as the level with which we compared the other plants, because

240 of its low cardenolide content. Eclosion success or failure was analysed with a binomial

241 GLM. To analyse how cardenolides and oxidative stress affected colour metrics in the

242 butterflies we used two methods. First, we used the R drop1 function to remove single model

243 terms to find the minimal adequate model and compared models using AIC (see

244 supplementary tables s1-s11). We explored interactions between two continuous variables

245 using the coplot function in the R package car [49]. Second, we built a generalized linear

246 model including six covariates a priori expected to affect warning signals (three markers of

247 oxidative state and three measures of sequestration - body, wing, and total CG content). The

248 model response variables were conspicuousness, redness, or brightness. We standardized all 
covariates to a mean of 0 and a standard deviation (SD) of 1 to facilitate comparisons between models and variables. We ran all possible models containing each covariate (without interactions) using the R package MuMIn [50] and summed the cumulative AIC support for the models containing each covariate. MDA, body cardenolides, and total cardenolides had AIC weights of $0.153,0.081$, and 0.075 (supplementary table s8). All data were analysed using R version 4.0.4.

\section{Results}

In support of our first hypothesis, concentrations of cardenolides in the body and wings of newly eclosed adults differed according to which host-plant species they were reared on (Figure 1a; $\left.\mathrm{F}_{(3,37)}=8.262, \mathrm{P}=0.0002\right)$. Monarchs reared on $A c$ and $A s$ sequestered significantly more cardenolides in total than those reared on $A t($ estimate $=822.9 \pm 184.3, \mathrm{t}=$ 4.47, $\mathrm{P}=7.6 \mathrm{e}-05$; estimate $=726.6 \pm 187.1, \mathrm{t}=3.88, \mathrm{P}=0.0004)$. Those reared on $\mathrm{Ai}$ did not sequester significantly different total amounts of CGs to those on $A t$ (estimate $=213.9 \pm$ 189.2, $\mathrm{t}=1.13, \mathrm{P}=0.27)$. Males tended to sequester more cardenolides in total than females, but this was not significant at the alpha 0.05 level (estimate $=243.5 \pm 133.4, \mathrm{t}=1.825, \mathrm{P}=$ 0.076).

Body CG concentration tended to differ between food plants, but this was not significant at significantly varied between host-plants (Figure $1 \mathrm{c} ; \mathrm{F}_{(3,37)}=26.23, \mathrm{P}<0.001$; Fig. 1c). Wing

269 CGs were significantly higher in $A \mathrm{c}$ and $A s$ compared to At (estimate $=1.56 \pm 0.27, \mathrm{t}=$

271 significantly between $A t$ and $A i(\mathrm{p}=0.38)$, nor between $A s$ and $A c(\mathrm{p}=0.79)$. 
273 We predicted that sequestration of cardenolides would impose a cost that would be associated

274 with reduced survivorship to eclosion. Successful eclosion was significantly higher in 275 monarchs reared on $A t(89 \%)$ compared with those reared on $A s(20 \%$; estimate $=-2.06 \pm$

$2760.751, \mathrm{t}=2.75, \mathrm{P}=0.009)$, while there was no significant difference between $\mathrm{Ai}(70 \%)$ and 277 At $(89 \%$, estimate $=-0.696 \pm 0.728, \mathrm{t}=-0.956, \mathrm{P}=0.34)$ or $\operatorname{At}(89 \%)$ and $A c(62 \%$; estimate $278=-0.927 \pm 0.690, \mathrm{t}=-1.344, \mathrm{P}=0.19)$. Survival to pupation did not differ significantly 279 according to host-plant species $(0 \% A t, 23 \% A i, 33 \% A s, 27 \% A c$; Fisher's exact test $\mathrm{P}=$ $280 \quad 0.28)$.

$281 \quad C G$ and oxidative stress

Malondialdehyde (MDA)

283 Contrary to our prediction that oxidative lipid damage would differ by hostplant, we found

284 that the minimal adequate model did not include species of host-plant, and only included sex

285 and the total concentration of cardenolide. In line with our prediction of a positive association between concentration of sequestered CGs and markers of oxidative damage, across all treatment groups individuals that sequestered higher total concentrations of CGs had higher levels of MDA (estimate $=0.0005 \pm 0.0002, \mathrm{t}=2.51, \mathrm{P}=0.016)$. There was no significant difference between the sexes (estimate $=-0.3097 \pm 0.198, \mathrm{t}=-1.57, \mathrm{P}=0.13$ ).

291 The minimal adequate model included only body CG concentration, which had no

292 statistically significant association with $\operatorname{SOD}($ estimate $=0.933 \pm 0.616, \mathrm{t}=1.51, \mathrm{P}=0.14$ ).

293 Total antioxidant capacity (TAC)

294 The minimal adequate model included foodplant, which did not significantly affect the concentrations of total antioxidant capacity $\left(\mathrm{TAC}: \mathrm{F}_{(3,38)}=0.3203, \mathrm{P}=0.81\right.$ ). 
Sequestration, oxidative stress and warning signals

297 Because malondialdehyde was the only marker associated with individual levels of

298 sequestration, we proceeded to analyse its association with warning signals, but did not

299 conduct analyses on superoxide dismutase activity or total antioxidant capacity effects on

300 warning signals. We found a significant three-way interaction between MDA x total CG

301 concentration $\mathrm{x}$ sex on the conspicuousness (chroma $\Delta \mathrm{S}$ ) of monarch forewings (estimate = -

$3020.009 \pm 0.005, \mathrm{t}=-2.05, \mathrm{P}=0.049)$. Because males were more conspicuous than females, we

303 separated the analysis by sex. We found a significant two way-interaction between MDA and

304 total $\mathrm{CG}$ concentration on the conspicuousness (chroma $\Delta \mathrm{S}$ ) of male forewings (estimate = -

$3050.006 \pm 0.002, \mathrm{t}=-2.98, \mathrm{P}=0.007$ ) that is represented by a curved surface (Figure 3 ). Males

306 with the highest levels of sequestered cardenolides had the most conspicuous warning signals

307 when oxidative damage was lowest, and reduced their warning signal conspicuousness as

308 oxidative damage increased, while those that sequestered the lowest levels of cardenolides

309 invested equally in warning signal conspicuous with increasing oxidative damage. There was

310 no significant effect of oxidative damage or concentration of sequestered cardenolides on

311 female forewing conspicuousness $($ MDA estimate $=0.320 \pm 2.7859, \mathrm{t}=0.12, \mathrm{P}=0.911 ; \mathrm{CG}$

312 total estimate $=-0.0005 \pm 0.003, \mathrm{t}=-0.14, \mathrm{P}=0.895)$. Males had significantly redder

313 forewings than females (estimate $=0.263 \pm 0.063, \mathrm{t}=4.15, \mathrm{P}=0.0002$ ), and as the

314 concentration of sequestered cardenolides increased the redness of the forewings increased

315 for both males and females, but this was not significant at the alpha 0.05 level (estimate $=$

$3160.0007 \pm 0.0003, \mathrm{t}=1.95, \mathrm{P}=0.059)$. Monarchs with low levels of oxidative damage

317 produced redder warning signals with increasing concentrations of sequestered cardenolides,

318 whereas and monarchs with high levels of oxidative damage produced less red warning

319 signals with increasing concentrations of sequestered cardenolides, but this was also not

320 significant at the alpha 0.05 level (MDA x total CG interaction: estimate $=-0.0002 \pm$ 
$3210.00009, \mathrm{t}=-1.864, \mathrm{P}=0.071)$. Males had significantly brighter forewings than females

322 (estimate $=0.027 \pm 0.007, \mathrm{t}=4.101, \mathrm{P}=0.0002$ ). Both male and female monarchs with

323 higher levels of MDA had significantly less bright forewings (estimate $=-0.01 \pm 0.005, \mathrm{t}=$

$3242.04, \mathrm{P}=0.048)$.

325 Discussion

326 We reared individual monarch caterpillars on whole plants from single populations of four

327 milkweed species with varying phytochemistry [Asclepias tuberosa, A. incarnata, A.syriaca,

328 and A.curassavica; 51]. Monarchs that sequestered higher concentrations of cardenolides

329 experienced higher levels of oxidative damage than those that sequestered lower

330 concentrations (measured as the concentration of malondialdehyde; MDA). Although there is

331 some evidence that cardenolides can be a burden for monarch caterpillars [22, 52], our results

332 are some of the first to show physiological costs of sequestration for monarchs [see also 21,

333 39]. Male monarchs that sequestered the highest concentrations of cardenolides had the most

334 conspicuous warning signals when oxidative damage was lowest, and reduced their warning

335 signal conspicuousness as oxidative damage increased, while those that sequestered the

336 lowest levels of cardenolides invested equally in warning signal conspicuous with increasing

337 oxidative damage. Our results suggest that highly toxic prey reduce investment in signals

338 because they cannot bear the oxidative cost of investing highly in both traits. This is the first

339 evidence to support Blount et al's [16] resource allocation trade-off model, and shows that

340 variable access to resources can result in differential costs of signalling in monarch butterflies

$341[53]$.

342 Some authors [54] have rejected the idea that there can be a mechanistic link between

343 warning signals and chemical defences that involve intrinsic costs of production or

344 maintenance. However, condition-dependent expression of colourful signals can arise when

345 the underlying physiological pathways that produce colouration are dependent on the same 
core cellular processes $[16,55]$. We found stable levels of antioxidant defences (superoxidase

347 dismutase and total antioxidant capacity) but increased oxidative damage was associated with

348 reduced warning signal conspicuousness, which suggests that the monarchs that are better

349 able to control pro-oxidant levels can sequester more cardenolides and still invest in warning

350 signals [56]. Similar patterns are observed in Aristolochia-feeding pipevine swallowtails

351 butterflies (Battus philenor) that store nitrophenanthrenes and have significantly higher tissue

352 levels of carotenoids than related species which mimic them in coloration but do not store

353 toxins [57]. Similarly, the rank order of body concentrations of carotenoids in three species of

354 lepidoptera matches the rank order of dietary exposure to the prooxidant toxin

355 furanocoumarin (8-MOP) [Papilio polyxenes $>$ Spodoptera eridania $>$ Trichoplusia ni; 18].

356 Antioxidants are involved in detoxification processes [58] as well as pigment synthesis

357 pathways directly [59], or as cofactors of enzymes [60]. Our results allow us to speculate that

358 antioxidant availability has a role in the biochemistry underlying the variations in warning

359 colouration and toxicity in aposematic animals. However, warning colours are usually

360 regulated by more than one mechanism, and we suggest that this area of research warrants

361 further biochemical study.

362 It is likely that oxidative state depends on the combination of genetic, environmental and

363 gene by environment interactions that determine an individual's condition. Monarchs show

364 patterns of local adaptation to their hostplants [based on larval growth rate 39], and also show

365 GxE interactions in sequestration ability [23] which may reflect either a lack of evolutionary

366 history with different species of Asclepias, or a physiological trade-off in sequestration

367 ability. Our results suggest that populations of monarchs that are locally adapted to high

368 cardenolide milkweeds could be less subject to oxidative damage, either because they have

369 evolved higher antioxidant efficiency, or because they are able to accumulate more

370 antioxidants to provide enhanced antioxidant protection. Further research on this topic could 
have interesting implications for understanding host shifts and range expansions as well as our understanding of automimicry (the occurrence of palatable 'cheaters' in a chemically defended population). Whether automimicry in monarchs arises directly from a deficiency in an individual's capacity to cope with reactive oxygen species warrants further study [61].

The resource competition model [16] does not investigate or predict sex differences in resource limitation. However, Blount et al [62] found that ladybird females (the larger sex) are more susceptible to resource limitation than males, and hence more likely to signal honestly. In this study we found costs of signalling in male monarchs. Males are slightly larger than females and are the more active sex; this behavioural difference may increase their probability of detection and risk of predation, and also increase their sensitivity to oxidative damage. If predators are sensitive to the combined qualities of toxins and displays when they attack prey, this could mean they avoid highly conspicuous prey and reject highly defended but less conspicuous prey, and instead predate moderately defended and conspicuous individuals, this could maintain the signal variability we observe in males and in other aposematic prey. The relationship between visual signal strength, chemical defence, and predator response warrants further study.

387 Although we did not measure cardenolide content in milkweed plants, our results confirm previous research showing that the concentration of cardenolides in monarch butterflies varies depending on host-plant chemistry $[63,64]$, and follows a standard approach to using the natural between species and population variability in plant toxicity to test for costs of

391 sequestration $[21,37,51]$. However, it is methodologically difficult to separate the costs of 392 sequestration from other potential effects of plant allelochemicals [65]. Future work could focus on comparative phytochemical profiles to understand resource availability, and experiments that control nutritional content while manipulating toxin content. 
395 In conclusion, our results support the proposal that oxidative state can be a key physiological mechanism that links warning colours to sequestration costs [16], and that the contribution of

397 pigments to antioxidant activity for maintaining redox homeostasis is important [61]. Our

398 results show that specialist herbivores must balance the benefits of plant secondary

399 compounds for sequestration with the burden that these same compounds impose [though see

$40051,66]$. Future research should examine the possibly of natural selection for oxidative

401 capacity, and whether aposematism evolves in species that have high antioxidant capacity.

402 These studies could provide new theories for understanding the evolution of aposematism

403 when coloration and toxicity do not coevolve. Documenting the costs associated with using

404 secondary defences in natural systems is important for our understanding of the ecology and 405 evolution of aposematism.

\section{Acknowledgements}

408 We thank Prof. Linda Fink for her support in drafting the manuscript, Prof. Innes Cuthill for 409 the MATLAB code for visual modelling, and Prof. Anurag Agrawal for leaves of Ascelpias 410 syriaca. JDB was supported by a Royal Society University Research Fellowship. HMR was 411 supported by a Junior Research Fellowship from Churchill College, Cambridge, and by

412 funding from the Max Planck Society.

\section{Author contributions}

415 Jonathan D. Blount conceptualization, methodology, resources, data curation, writing -

416 original draft, project administration, funding acquisition.

417 Hannah M. Rowland methodology, formal analysis, investigation, data curation, writing 418 original draft, visualization. 
419 Christopher Mitchell investigation, data curation.

420 Michael P. Speed conceptualization, methodology, writing - review \& editing.

421 Graeme D. Ruxton conceptualization, methodology, writing - review \& editing.

422

John A. Endler conceptualization, writing - review \& editing, visualization.

423

Lincoln P. Brower conceptualization, methodology, investigation, resources.

424

425

\section{References}

426

427

[1] Blum, M.S. 1981 Chemical Defenses of Arthropods. New York, Academic Press.

428

[2] Ruxton, G.D., Allen, W.L., Sherratt, T.N. \& Speed, M.P. 2018 Avoiding Attack. The

429

Evolutionary Ecology of Crypsis, Aposematism, and Mimicry. Oxford, UK., Oxford

430

University Press.

431 [3] Summers, K. \& and Clough, M.E. 2001 The evolution of coloration and toxicity in the 432 poison frog family (Dendrobatidae). Proceedings of the National Academy of Sciences $\mathbf{9 8 ,}$ $4336227-6232$.

434 [4] Santos, J.C. \& Cannatella, D.C. 2011 Phenotypic integration emerges from aposematism 435 and scale in poison frogs. Proceedings of the National Academy of Sciences 108, 6175-6180. 436 (doi:10.1073/pnas.1010952108).

437 [5] Maan, M.E. \& Cummings, M.E. 2009 Sexual dimorphism and directional sexual selection 438 on aposematic signals in a poison frog. Proceedings of the National Academy of Sciences 439 106, 19072-19077. (doi:10.1073/pnas.0903327106).

440 [6] Cortesi, F. \& Cheney, K.L. 2010 Conspicuousness is correlated with toxicity in marine 441 opisthobranchs. Journal of Evolutionary Biology 23, 1509-1518. (doi:doi:10.1111/j.1420$442 \quad 9101.2010 .02018 . x$ ). 
443 [7] Bezzerides, A., L., McGraw, K.J., Parker, R.S. \& Husseini, J. 2007 Elytra color as a

444 signal of chemical defense in the Asian ladybird beetle Harmonia axyridis. Behavioral

445 Ecology and Sociobiology 61, 1401-1408. (doi:10.1007/s00265-007-0371-9).

446 [8] Winters, A.E., Stevens, M., Mitchell, C., Blomberg, S.P. \& Blount, J.D. 2014 Maternal 447 effects and warning signal honesty in eggs and offspring of an aposematic ladybird beetle. 448 Functional Ecology 28, 1187-1196. (doi:doi:10.1111/1365-2435.12266).

449 [9] Vidal-Cordero, J.M., Moreno-Rueda, G., López-Orta, A., Marfil-Daza, C., Ros-Santaella, 450 J.L. \& Ortiz-Sánchez, F.J. 2012 Brighter-colored paper wasps (Polistes dominula) have larger 451 poison glands. Frontiers in Zoology 9, 20. (doi:10.1186/1742-9994-9-20).

452 [10] Summers, K., Speed, M.P., Blount, J.D. \& Stuckert, A.M.M. 2015 Are aposematic 453 signals honest? A review. Journal of Evolutionary Biology, n/a-n/a. (doi:10.1111/jeb.12676).

454 [11] Leimar, O., Enquist, M. \& Sillentullberg, B. 1986 Evolutionary Stability of Aposematic 455 Coloration and Prey Unprofitability - a Theoretical-Analysis. American Naturalist 128, 469456490.

457 [12] Speed, M.P. \& Ruxton, G.D. 2007 How bright and how nasty: Explaining diversity in 458 warning signal strength. Evolution 61, 623-635.

459 [13] Ruxton, G.D., Speed, M.P. \& Broom, M. 2009 Identifying the ecological conditions that 460 select for intermediate levels of aposematic signalling. Evolutionary Ecology 23, 491-501. 461 (doi:10.1007/s10682-008-9247-3).

462 [14] Darst, C.R., Cummings, M.E. \& Cannatella, D.C. 2006 A mechanism for diversity in 463 warning signals: Conspicuousness versus toxicity in poison frogs. Proc. Natl. Acad. Sci. U. S. 464 A. 103, 5852-5857.

465 [15] Wang, I.J. 2011 Inversely related aposematic traits: reduced conspicuousness evolves 466 with increased toxicity in a polymorphic poison-dart frog. Evolution 65, 1637-1649. 467 (doi:doi:10.1111/j.1558-5646.2011.01257.x). 
468

469

470

471

472

473

474

475

476

477

478

479

480

481

482

483

484

485

486

487

488

489

490

491

492

[16] Blount, J.D., Speed, M.P., Ruxton, G.D. \& Stephens, P.A. 2009 Warning displays may function as honest signals of toxicity871-877 p.

[17] Holloway, G.J., de Jong, P.W., Brakefield, P.M. \& de Vos, H. (1991) Chemical defence in ladybird beetles (Coccinellidae). I. Distribution of coccinelline and individual variation in defence in 7-spot ladybirds (Coccinella septempunctata). Chemoecology, 2, 7-14.

[18] Ahmad, S. 1992 Biochemical defence of pro-oxidant plant allelochemicals by herbivorous insects. . Biochemical Systematics and Ecology 20, 269-296.

[19] Holen, O.H. \& Svennungsen, T.O. 2012 Aposematism and the Handicap Principle. The American Naturalist 180, 629-641. (doi:10.1086/667890).

[20] Malcolm, S.B. \& Brower, L.P. 1989 Evolutionary and Ecological Implications of Cardenolide Sequestration in the Monarch Butterfly. Experientia 45, 284-295.

[21] Agrawal, A.A., Böröczky, K., Haribal, M., Hastings, A.P., White, R.A., Jiang, R. \& Duplais, C. 2021 Cardenolides, toxicity, and the costs of sequestration in the coevolutionary interaction between monarchs and milkweeds. Proceedings of the National Academy of Sciences 118, e2024463118. (doi:10.1073/pnas.2024463118).

[22] Tao, L., Berns, A.R. \& Hunter, M.D. 2014 Why does a good thing become too much? Interactions between foliar nutrients and toxins determine performance of an insect herbivore. Functional Ecology 28, 190-196. (doi:https://doi.org/10.1111/1365-2435.12163).

[23] Freedman, M.G., Choquette, S.-L., Ramírez, S.R., Strauss, S.Y., Hunter, M.D. \& Vannette, R.L. 2021 Population-specific patterns of toxin sequestration in monarch butterflies from around the world. bioRxiv, 2021.2010.2015.464593. (doi:10.1101/2021.10.15.464593).

[24] Brower, L.P. 1969 Ecological chemistry. Scientific American 220, 22-29.

[25] Brower, L.P. 1972 Ecological Chemistry of Palatability Cardiac Glycoside Spectrum in Monarch Butterflies, Danaus-Plexippus, and Asclepias Milkweeds. American Zoologist 12, 712-713. 
493 [26] Züst, T., Petschenka, G., Hastings, A.P. \& Agrawal, A.A. 2019 Toxicity of Milkweed

494 Leaves and Latex: Chromatographic Quantification Versus Biological Activity of

495 Cardenolides in 16 Asclepias Species. J Chem Ecol 45, 50-60. (doi:10.1007/s10886-018-

496 1040-3).

497 [27] Tao, L., Hoang, K.M., Hunter, M.D. \& de Roode, J.C. 2016 Fitness costs of animal 498 medication: antiparasitic plant chemicals reduce fitness of monarch butterfly hosts. J Anim 499 Ecol 85, 1246-1254. (doi:10.1111/1365-2656.12558).

500 [28] Groen, S.C., LaPlante, E.R., Alexandre, N.M., Agrawal, A.A., Dobler, S. \& Whiteman, 501 N.K. 2017 Multidrug transporters and organic anion transporting polypeptides protect insects 502 against the toxic effects of cardenolides. Insect Biochemistry and Molecular Biology 81, 5150361.

504 [29] Seiber, J.N., Tuskes, P.M., Brower, L.P. \& Nelson, C.J. 1980 Pharmacodynamics of 505 Some Individual Milkweed Cardenolides Fed to Larvae of the Monarch Butterfly (Danaus506 Plexippus L). Journal of Chemical Ecology 6, 321-339.

507 [30] Roeske, C.N., Seiber, J.N., Brower, L.P. \& Moffitt, C.M. 1976 Milkweed Cardenolides 508 and Their Comparative Processing by Monarch Butterflies (Danaus plexippus L.). In 509 Biochemical Interaction Between Plants and Insects (eds. J.W. Wallace \& R.L. Mansell), pp. 510 93-167. Boston, MA, Springer US.

511 [31] Faldyn, M.J., Hunter, M.D. \& Elderd, B.D. 2018 Climate change and an invasive, 512 tropical milkweed: an ecological trap for monarch butterflies. Ecology 99, 1031-1038. 513 (doi:https://doi.org/10.1002/ecy.2198).

514 [32] Agrawal, A.A. 2017 Monarchs and Milkweed: A Migrating Butterfly, a Poisonous Plant, 515 and their Remarkable Story of Coevolution Princeton, NJ., Princeton University Press.

516 [33] Geest, E.A., Wolfenbarger, L.L. \& McCarty, J.P. 2019 Recruitment, survival, and 517 parasitism of monarch butterflies (Danaus plexippus) in milkweed gardens and conservation 518 areas. Journal of Insect Conservation 23, 211-224. (doi:10.1007/s10841-018-0102-8). 
519 [34] Malcolm, S.B., Cockrell, B.J. \& Brower, L.P. 1989 Cardenolide Fingerprint of Monarch

520 Butterflies Reared on Common Milkweed, Asclepias-Syriaca-L. Journal of Chemical

521 Ecology 15, 819-853.

[35] Petschenka, G. \& Agrawal, A.A. 2015 Milkweed butterfly resistance to plant toxins is linked to sequestration, not coping with a toxic diet. Proceedings. Biological sciences / The Royal Society 282, 20151865. (doi:10.1098/rspb.2015.1865).

[36] Brower, L.P. 1970 Plant poisons in a terrestrial food chain and implications for mimicry

526 theory. . In Biochemical Coevolution (Proceedings of the Twenty-Ninth Annual Biology

527 Colloquium). (ed. K.L. Chambers), pp. 69-82. Corvallis, Oregon., Oregon State University 528 Press, .

529 [37] Soule, A.J., Decker, L.E. \& Hunter, M.D. 2020 Effects of diet and temperature on 530 monarch butterfly wing morphology and flight ability. Journal of Insect Conservation 24, 531 961-975. (doi:10.1007/s10841-020-00267-7).

532 [38] Woods, E.C., Hastings, A.P., Turley, N.E., Heard, S.B. \& Agrawal, A.A. 2012 Adaptive 533 geographical clines in the growth and defense of a native plant. Ecological Monographs 82, 149-168. (doi:https://doi.org/10.1890/11-1446.1). (doi:https://doi.org/10.1111/evo.13914). L.P. 2017 Milkweed Matters: Monarch Butterfly (Lepidoptera: Nymphalidae) Survival and

540 Development on Nine Midwestern Milkweed Species. Environ. Entomol. 46, 1098-1105.

541 (doi:10.1093/ee/nvx137).

542 [41] Malcolm, S.B. \& Zalucki, M.P. 1996 Milkweed latex and cardenolide induction may 543 resolve the lethal plant defence paradox. In Proceedings of the 9th International Symposium 544 on Insect-Plant Relationships (eds. E. Städler, M. Rowell-Rahier \& R. Bauer), pp. 193-196.

545 Dordrecht, Springer Netherlands. 
546 [42] Rasmann, S., Agrawal, A.A., Cook, S.C. \& Erwin, A.C. 2009 Cardenolides, induced

547 responses, and interactions between above- and belowground herbivores of milkweed

548 (Asclepias spp.). Ecology 90, 2393-2404. (doi:https://doi.org/10.1890/08-1895.1).

549 [43] Fink, L.S., Brower, L.P., Waide, R.B. \& Spitzer, P.R. 1983 Overwintering Monarch

550 Butterflies as Food for Insectivorous Birds in Mexico. Biotropica 15, 151-153.

551 [44] Vorobyev, M. \& Osorio, D. 1998 Receptor noise as a determinant of colour thresholds.

552 Proc. R. Soc. Lond. Ser. B-Biol. Sci. 265, 351-358.

553 [45] Keyser, A.J. \& Hill, G.E. 2000 Structurally based plumage coloration is an honest signal 554 of quality in male blue grosbeaks. Behavioral Ecology 11, 202-209.

555 [46] Hart, N.S., Partridge, J.C., Cuthill, I.C. \& Bennett, A.T.D. 2000 Visual pigments, oil 556 droplets, ocular media and cone photoreceptor distribution in two species of passerine bird: 557 the blue tit (Parus caeruleus L.) and the blackbird (Turdus merula L.). J. Comp. Physiol. A558 Sens. Neural Behav. Physiol. 186, 375-387.

559 [47] Stevens, M. \& Merilaita, S. 2011 Animal Camouflage: Mechanisms and Function.

560 Cambridge, UK, Cambridge University Press.

561 [48] Osorio, D. \& Vorobyev, M. 2005 Photoreceptor spectral sensitivities in terrestrial 562 animals: adaptations for luminance and colour vision. Proceedings of the Royal Society of 563 London, Series B 272, 1745-1752.

564 [49] Fox, J. \& Weisberg, S. 2019 An R Companion to Applied Regression. Third edition. ed, 565 Sage, Thousand Oaks CA.

566 [50] Bartoń, K. 2020 MuMIn: Multi-Model Inference. (

567 [51] Erickson, J.M. 1973 The Utilization of Various $<\mathrm{em}>$ Asclepias $</ \mathrm{em}>$ Species by Larvae 568 of the Monarch Butterfly, $<\mathrm{em}>$ Danaus Plexippus $</ \mathrm{em}>$. Psyche 80, 028693.

569 (doi:10.1155/1973/28693). 
[52] Zalucki, M.P., Brower, L.P. \& Alonso, A. 2001 Detrimental effects of latex and cardiac glycosides on survival and growth of first-instar monarch butterfly larvae Danaus plexippus feeding on the sandhill milkweed Asclepias humistrata. Ecological Entomology 26, 212-224. puzzle. Oxford, UK, Oxford University Press.

[55] Hill, G.E. 2011 Condition-dependent traits as signals of the functionality of vital cellular

[56] Costantini, D., Fanfani, A. \& Dell'Omo, G. 2008 Effects of corticosteroids on oxidative damage and circulating carotenoids in captive adult kestrels (Falco tinnunculus). Journal of Comparative Physiology B 178, 829-835. (doi:10.1007/s00360-008-0270-z). product reports, 18, 310-333. insecticide resistance. Insect Mol Biol, 14, 3-8. tyrosinase. Experientia, 33, 1118-1119. biosynthesis of insect pigments. EJE, 111, 149-164.

590 [61] Costantini, D. \& Verhulst, S. 2009 Does high antioxidant capacity indicate low oxidative 591 stress? Functional Ecology 23, 506-509. (doi:https://doi.org/10.1111/j.1365$592 \quad 2435.2009 .01546 . x)$.

593 [62] Blount, J.D., Rowland, H.M., Drijfhout, F.P., Endler, J.A., Inger, R., Sloggett, J.J., 594 Hurst, G.D.D., Hodgson, D.J. \& Speed, M.P. 2012 How the ladybird got its spots: effects of 
resource limitation on the honesty of aposematic signals. Functional Ecology 26, 334-342. (doi:10.1111/j.1365-2435.2012.01961.x).

597

[63] Jones, P.L., Petschenka, G., Flacht, L. \& Agrawal, A.A. 2019 Cardenolide Intake,

598 Sequestration, and Excretion by the Monarch Butterfly along Gradients of Plant Toxicity and Larval Ontogeny. Journal of Chemical Ecology 45, 264-277. (doi:10.1007/s10886-019-

$600 \quad 01055-7)$.

601 [64] Brower, L.P., Nelson, C.J., Seiber, J.N., Fink, L.S. \& Bond, C. 1988 Exaptation as an 602 alternative to coevolution in the cardenolide-based chemical defense of monarch butterflies

603 (Danaus plexippus L.) against avian predators. In Chemical mediation of coevolution (ed.

604 K.C. Spencer). London, Harcourt Brace Jovanovich.

605 [65] Bowers, M.D. 1992 The evolution of unpalatability and the cost of chemical defense in 606 insects. . In Insect chemical ecology: an evolutionary approach. Chapman \& Hall, New York, 607 New York, USA. (eds. B.D. Roitberg \& M.B. Isman), pp. 216-244.

608 [66] Petschenka, G. \& Agrawal, A.A. 2015 Milkweed butterfly resistance to plant toxins is 609 linked to sequestration, not coping with a toxic diet. Proceedings of the Royal Society B:

610 Biological Sciences 282. (doi:10.1098/rspb.2015.1865).

611

612 Figure 1. cardenolide sequestration by monarchs feeding on Ascelpias tuberosa (AT), A.

613 Incarnata (AI), A. Syriaca (AS), and A. curassavica (AC); a) total cardenolide ( $\mu$ g total

614 cardenolides per $0.1 \mathrm{~g}$ tissue) measured in the wings and bodies, b) body cardenolide content,

615 c) wing cardenolide content. Boxplots show the median, interquartile range, and the whiskers

616 represent the largest and smallest value within 1.5 times the $25^{\text {th }}$ and $75^{\text {th }}$ percentile. Outliers

617 are represented by small black dots. The mean cardenolide concentration is represented by

618 the larger black dot. 
619 Figure 2. Total cardenolide concentration for males (filled triangle) and females (open circle)

620 and Malondialdehyde (MDA) concentration. Line is the smoothed conditional mean with

$62195 \%$ confidence intervals.

622 Figure 3. Three-dimensional surface (perspective) plot for the response surface predicting

623 the relationship between the conspicuousness of the male monarch's forewing, oxidative

624 damage measured as malondialdehyde (MDA), and total cardenolide concentration. 

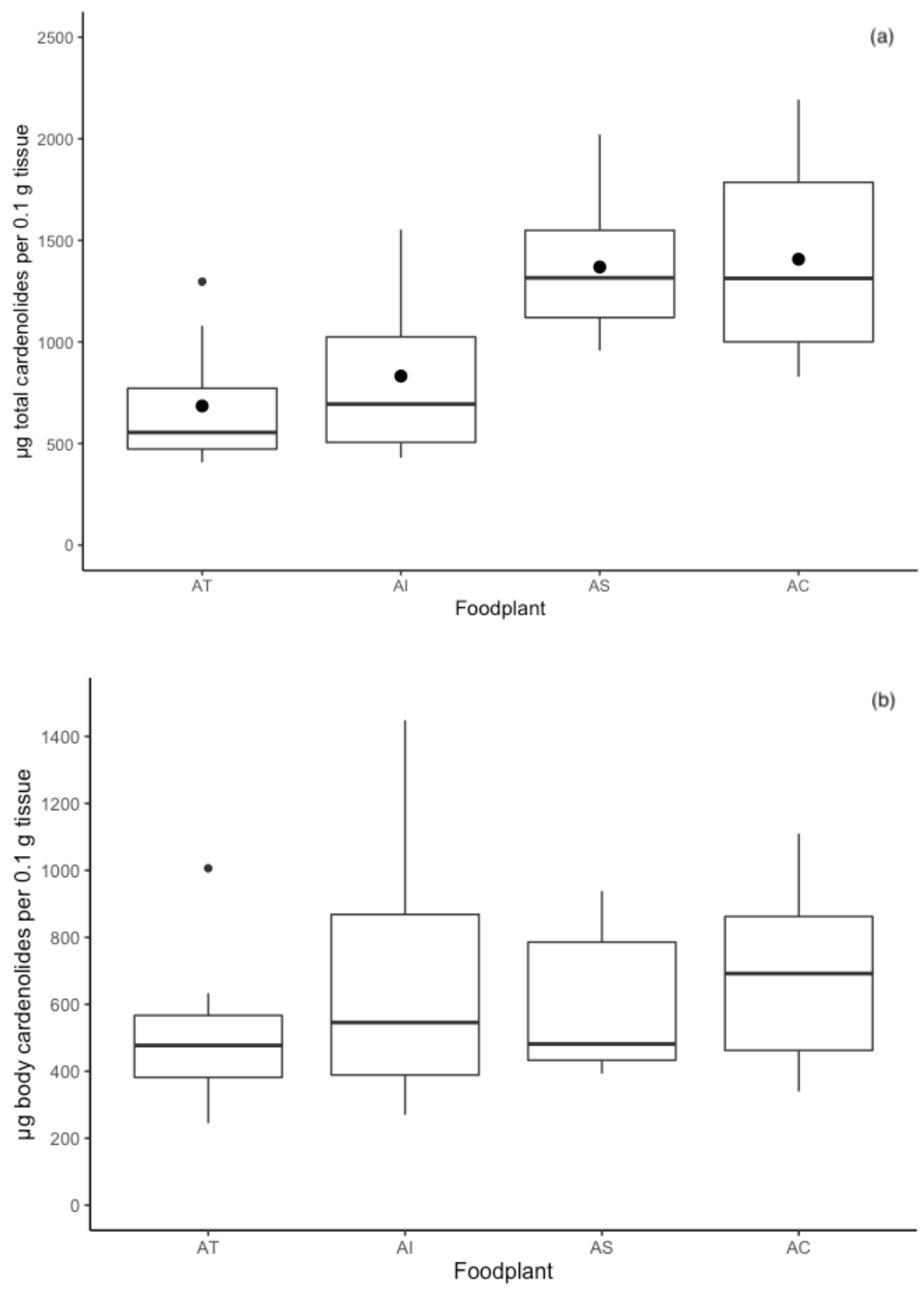


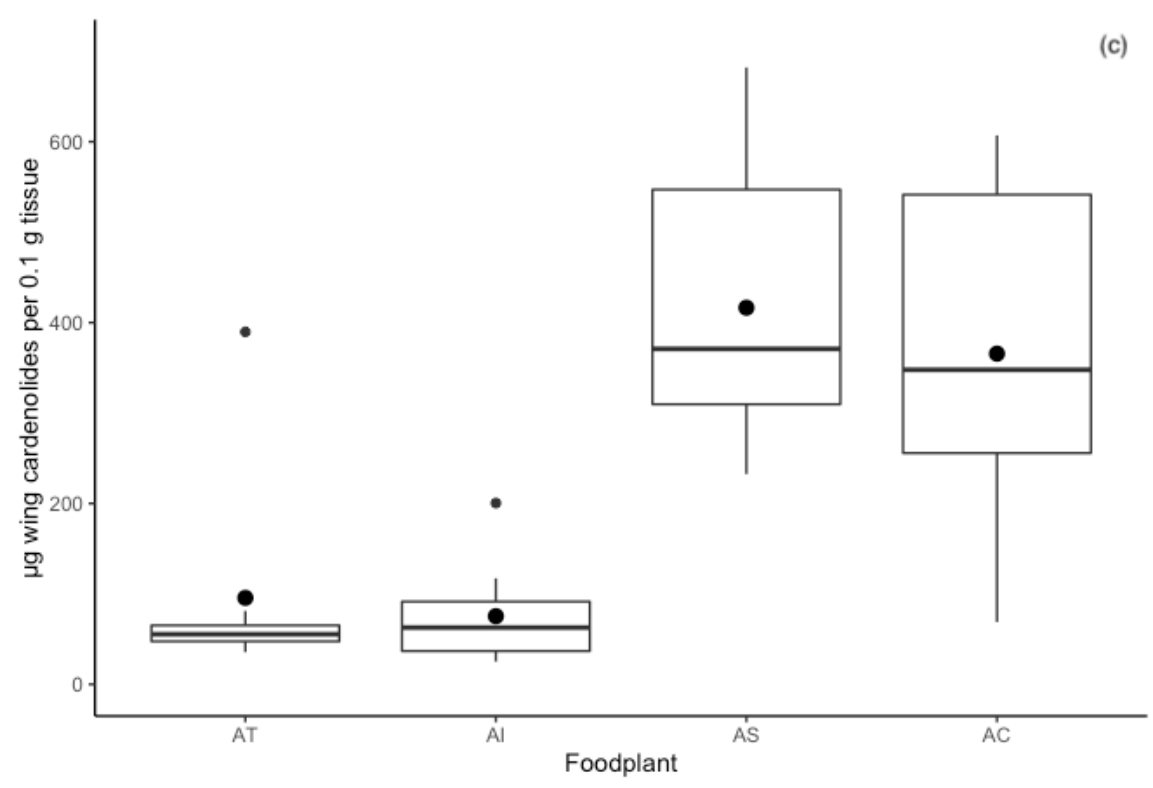

628 Figure 1a, b, c.

629

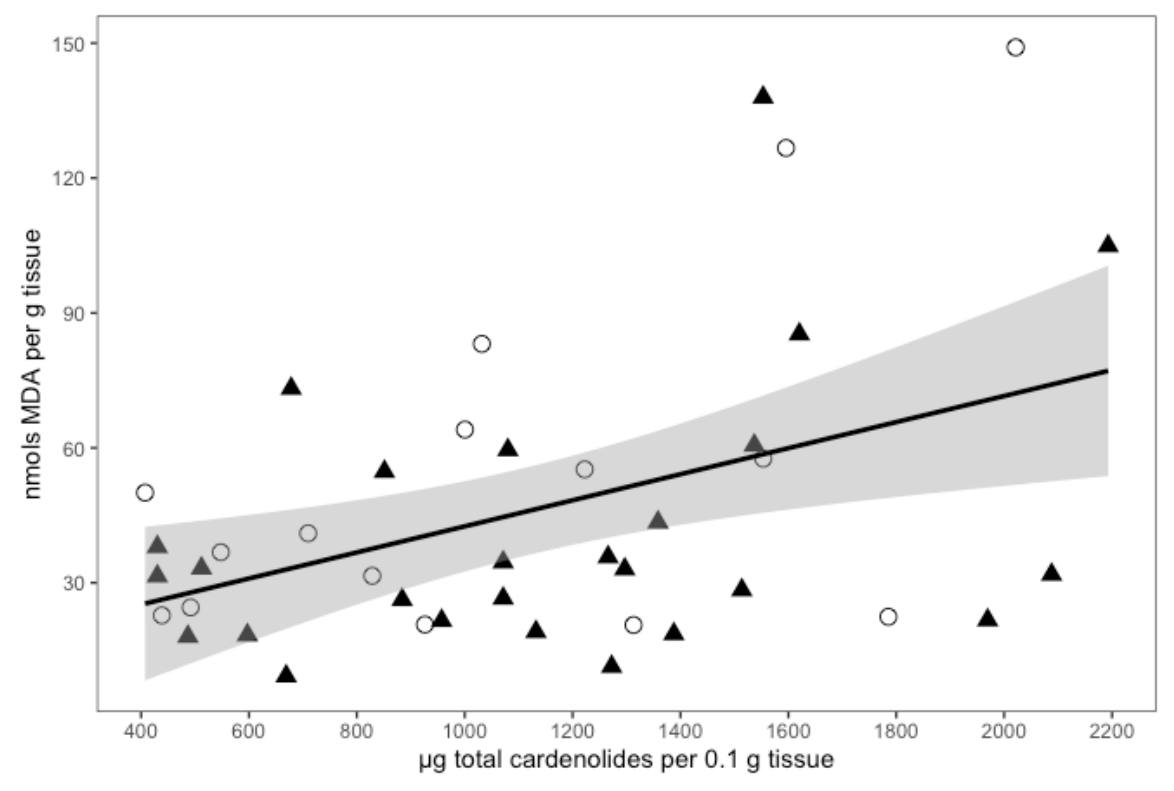

630

Figure 2 


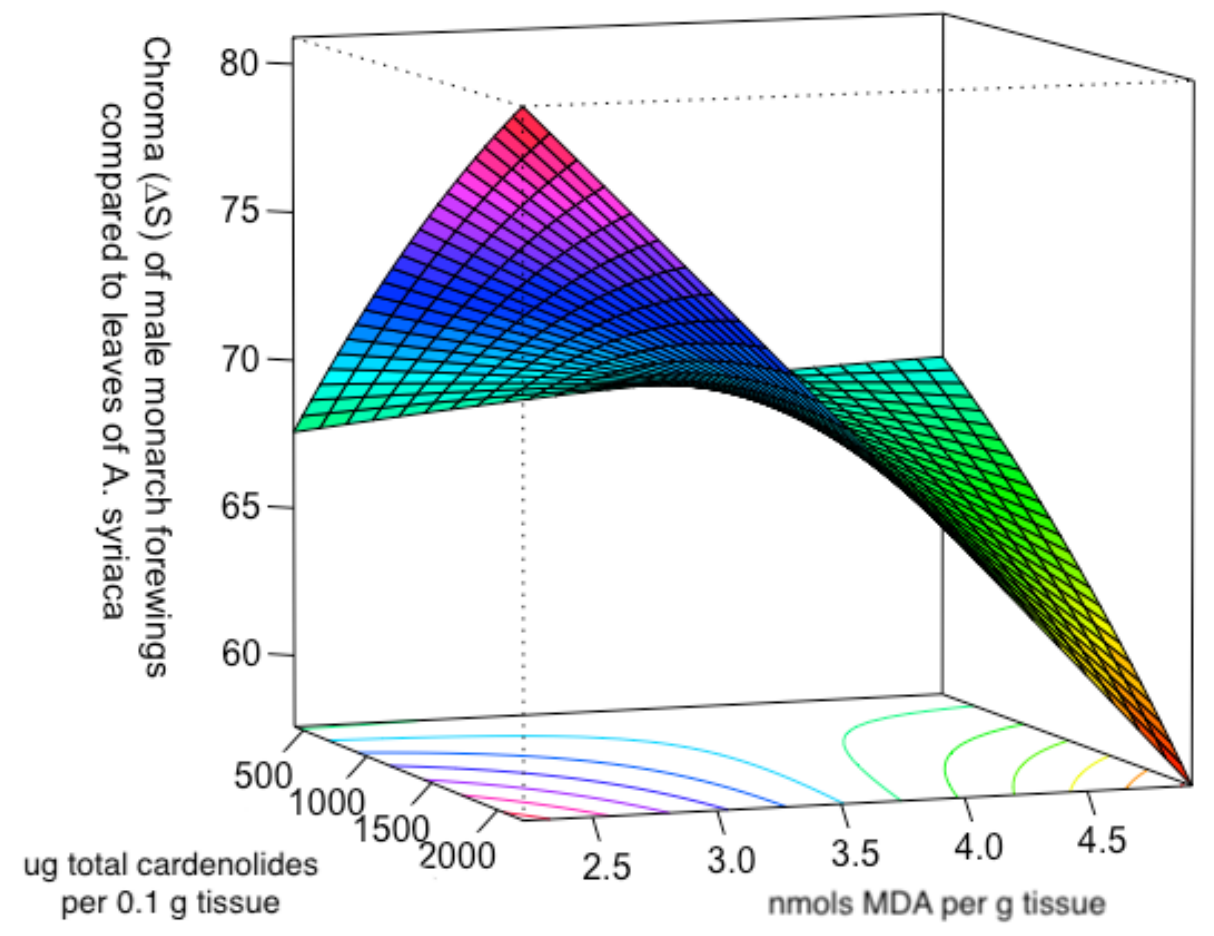

631

$632 \quad$ Figure 3 